\section{El efecto placebo en los estudios sobre la depresión mayor}

Los aspectos éticos de la administración de placebo en los ensayos clínicos siguen siendo tema de controversia. Los estudios sobre los tratamientos farmacológicos de la depresión mayor (DM) han consistido tradicionalmente en ensayos clínicos aleatorizados controlados con placebo y ha sido sobre esta base que se ha aprobado la comercialización de los fármacos antidepresivos, que se encuentran entre las medicaciones más utilizadas en todo el mundo. Varios investigadores han señalado que en los últimos años se ha hecho cada vez más difícil demostrar efectos antidepresivos específicos en comparación con el placebo, posiblemente porque la respuesta a este haya aumentado. En este estudio los autores examinaron los datos sobre la respuesta al placebo y a la medicación obtenidos en ensayos clínicos controlados de pacientes ambulatorios adultos con DM, para determinar si las características de dichos estudios y la respuesta al placebo han cambiado.

Se incluyeron los estudios publicados entre 1981 y 2000. Los publicados entre 1981 y 1990 procedieron en su mayoría de una amplia revisión de la literatura publicada anteriormente, y los publicados entre 1991 y 2000 se identificaron mediante una búsqueda bibliográfica en las bases de datos MEDLINE y PsycLit, utilizando como claves las palabras "placebo" y los nombres farmacológicos de todos los antidepresivos. Para ser incluidos en esta revisión, los estudios tenían que cumplir los siguientes requisitos: ser ensayos clínicos aleatorizados, doblemente enmascarados, controlados con placebo; haber sido publicados en inglés, entre enero de 1981 y enero de 2000; incluir fundamentalmente a pacientes ambulatorios con DM diagnosticada según los Criterios Diagnósticos de Investigación (RDC), los criterios del Manual Diagnóstico y Estadístico de las Enfermedades Mentales (DSM), versiones DSM-III, DSM-IIIR o DSM-IV, o los criterios de la Clasificación Internacional de Enfermedades, versiones CIE-9 o CIE-10; tener al menos 20 pacientes en el grupo tratado con placebo; tener una duración mínima de 4 semanas, y describir el número de pacientes asignados a un tratamiento $\mathrm{u}$ otro y el número de los que respondieron. La respuesta se definió como una reducción de $50 \%$ o más en la puntuación de la Escala de Depresión de
Hamilton (EDH) o como una mejoría marcada o moderada (puntuación de 1 a 2) en la Impresión Clínica Global (CGI).

Se identificaron 75 estudios que cumplían estos criterios. Su duración media ( \pm desviación estándar) fue de $45,4( \pm 17,9)$ días (recorrido: 28 a 168$)$ y aumentó significativamente con el año de publicación. El número medio de pacientes fue de 83,8 $( \pm 59,7)$ (recorrido: 20 a 336) en el grupo tratado con placebo y $85,0( \pm 60,0)$ (recorrido: 20 a 335$)$ en el tratado con fármacos activos; ninguna de estas dos cifras cambió de forma significativa con el año de publicación. Como era de esperar, los sistemas diagnósticos cambiaron con el tiempo. Por ejemplo, la proporción de estudios que utilizaron los criterios RDC disminuyó de 28,6\% entre 1981 y 1985 a $0 \%$ entre 1996 y 2000. En la mayoría de los estudios $(74,7 \%)$ hubo una fase preliminar de tratamiento con placebo y los pacientes que respondieron en esta fase no fueron aleatorizados; el número de estudios que emplearon este diseño no cambió significativamente a lo largo del tiempo. La puntuación mínima en la EDH requerida para que los pacientes fueran aceptados fue de 16,7 $( \pm 2,1)$ (recorrido: 12,9 a 22,0$)$ y aumentó con el año de publicación. Las dos clases de medicaciones más estudiadas fueron los antidepresivos tricíclicos (ADT, 43 estudios) y los inhibidores selectivos de la recaptación de serotonina (ISRS, 33 estudios).

Por término medio, $60,1 \%( \pm 9,7 \%)$ (recorrido: 36,2 a $85,0 \%$ ) de los participantes fueron mujeres, proporción que no cambió de forma significativa a lo largo del tiempo. La media de edad fue de 42,6 ( \pm 7,3 ) años (recorrido: 35,5 a 72,0) y aumentó significativamente con el año de publicación. La puntuación media de la EDH al inicio del estudio fue de $22,5( \pm 2,7)$ (recorrido: 13,8 a 30,2$)$ y no cambió significativamente a lo largo del tiempo.

Definiendo la respuesta como una reducción de la puntuación de la EDH en $50 \%$ o más (53 estudios), la tasa de respuesta al placebo (TRP) fue de $30,0 \%( \pm 8,0 \%)$; definiéndola como una puntuación de 1 a 2 en la CGI (47 estudios), fue de 32,8\% $( \pm 11,1 \%)$. En ambos casos, la cifra aumentó significativamente con el año de publicación. Combinando los datos obtenidos con ambos tipos de medidas, la TRP fue de $29,7 \%( \pm 8,3 \%)$ (recorrido: 12,5 a $51,8 \%$ ) y también se correlacionó de forma significativa con el año de publicación, así como con la duración del estudio y la puntuación inicial mínima 
requerida en la $\mathrm{EDH}$. Sin embargo, en un modelo de regresión lineal múltiple, solo siguió siendo significativa la correlación con el año de publicación.

La proporción media de pacientes que respondieron a la medicación fue de $50,1 \%( \pm 9,0 \%)$ (recorrido: 31,6 a $70,4 \%): 46,9 \%( \pm 10,6 \%)$ (recorrido: $27,5$ a $65,6 \%)$ para los ADT y $48,9 \%( \pm 10,3 \%$ ) (recorrido: 25,0 a 70,4\%) para los ISRS. Dicha proporción también se correlacionó con el año de publicación, pero al incluir las otras covariables en los modelos de regresión, todas las relaciones, tanto con el año de publicación como con las otras covariables, dejaron de ser estadísticamente significativas. El tamaño del efecto, esto es, la diferencia entre la respuesta a la medicación y la respuesta al placebo, fue, por término medio, de 0,43 $( \pm 0,22)$ : 0,38 $( \pm 0,22)$ para los ADT y $0,40( \pm 0,24)$ para los ISRS. Ninguna de estas tres magnitudes del efecto se correlacionó de forma estadísticamente significativa con el año de publicación.

El presente estudio reveló, en primer lugar, que la TRP es variable según los estudios, oscilando entre 10 y $50 \%$, aproximadamente; en cerca de la mitad de los estudios fue considerable: de $30 \%$ o más. En segundo lugar se observó que la TRP ha aumentado a lo largo de las dos últimas décadas, a una velocidad de aproximadamente $7 \%$ por década, y que la proporción de pacientes que responden a la medicación ha sufrido un aumento similar. El tamaño del efecto no ha cambiado de forma significativa en los estudios publicados, lo cual destaca la importancia de examinar los datos no publicados para determinar si el aumento de la TRP puede haber contribuido a la dificultad para establecer la eficacia de los nuevos antidepresivos.

Esta revisión revela que la respuesta al placebo en los ensayos clínicos controlados sobre los antidepresivos es muy variable, pero a menudo de magnitud considerable, y que ha aumentado en los últimos años, al igual que la respuesta a la medicación. Estos datos respaldan la importancia científica de la inclusión de un grupo tratado con placebo en los ensayos clínicos sobre los antidepresivos, lo cual hace necesario seguir reduciendo los potenciales riesgos de dichos estudios, de forma que se puedan realizar de un modo aceptable desde el punto de vista ético. El aumento de la TRP no parece ser atribuible directamente a cambios en las características de los estudios. Aunque los sistemas diagnósticos utilizados han ido cambiando con el tiempo, todos ellos están muy relacionados con los RDC, por lo cual no hay motivos para pensar que pueda ser esta la explicación para los cambios de la TRP. La edad media de los pacientes ha aumentado, pero la relación entre la edad y la TRP no fue significativa. La duración de los ensayos clínicos ha aumentado, correlacionándose directamente con el aumento de la TRP, hecho que posiblemente se deba tanto a los efectos acumulativos de las intervenciones inespecíficas inherentes a los ensayos clínicos como al aumento del período de tiempo durante el cual se puede producir una recuperación espontánea. También es de destacar el hecho de que la TRP media fuera similar en los estudios con y sin una fase preliminar de tratamiento con placebo. La mayor aceptación y uso de los antidepresivos puede haber aumentado la participación en los ensayos clínicos de pacientes con formas de depresión más leves, más breves $y$, por consiguiente, con mayor tendencia a responder al tratamiento, sea con fármacos activos o con placebo. Asimismo, hay que tener en cuenta que en la mayoría de los estudios se permitió el tratamiento simultáneo con sedantes o ansiolíticos, de modo que lo que se describe como una respuesta al placebo puede incluir los efectos de estos fármacos. (Walsh BT, Seidman SN, Sysko R, Gould M. Placebo response in studies of major depression: variable, substantial, and growing. JAMA 2002;287:1840-1847).

\section{Efecto de la prohibición de fumar en el lugar de trabajo sobre el comportamiento de los fumadores}

En los Estados Unidos de América se ha calculado que cada año mueren 53000 no fumadores por efecto del consumo pasivo. La prohibición de fumar en el lugar de trabajo tiene un importante efecto sobre el consumo de tabaco, como reconoce la propia industria tabacalera, que calcula que si estuviera prohibido fumar en todos los lugares de trabajo el consumo total disminuiría en cerca de $10 \%$. En este estudio los autores investigaron los efectos de la prohibición de fumar en el lugar de trabajo sobre el consumo de cigarrillos, y los compararon con los obtenidos por el aumento de los impuestos sobre los cigarrillos.

Se realizó una revisión sistemática de los estudios que han investigado los efectos de los lugares de trabajo totalmente libres de humo sobre la prevalencia del tabaquismo y el consumo diario de cigarrillos. Para ello se realizó una búsqueda bibliográfica en diferentes bases de datos (MEDLINE, Science Citation Index, Social Sciences Citation Index, Current Contents y PsychInfo), en revisiones sobre el tema y en las referencias de los artículos encontrados. Se incluyeron 26 estudios prospectivos, transversales o retrospectivos realizados en Alemania, Australia, Canadá y Estados Unidos. Se calcularon las diferencias en el consumo (por fumador y por empleado) y en la prevalencia antes y después de que se prohibiera fumar en el lugar de trabajo (en los estudios de lugares de trabajo) o entre muestras comparables con y sin regulación del consumo de 
tabaco (en los estudios poblacionales). Se realizó un metaanálisis de efectos aleatorios en el que se agruparon todos los estudios. Las pruebas realizadas no revelaron la existencia de sesgos de publicación.

La prohibición total de fumar en el lugar de trabajo se asoció a una reducción de la prevalencia absoluta en 3,8\% [intervalo de confianza del 95\% (IC95): $2,8 \%$ a $4,7 \%$ ] y a una disminución del consumo en 3,1 (IC95: 2,4 a 3,8) cigarrillos al día en las personas que siguieron fumando. Los efectos combinados del abandono del consumo y de la disminución del consumo por parte de los que siguieron fumando significó una disminución del consumo de 1,3 (recorrido: 0,2 a 1,8) cigarrilos por día y empleado (fumadores y no fumadores), lo cual corresponde a una reducción relativa de 29\% (IC95: 11 a $53 \%$ ). Estos efectos fueron estables a lo largo del tiempo. La prohibición total de fumar en el lugar de trabajo tuvo un efecto sobre la prevalencia y el consumo casi dos veces mayor que la prohibición parcial, esto es, la prohibición de fumar solo en algunas zonas del lugar de trabajo.

Por otra parte, el aumento de los impuestos sobre los cigarrillos en 10\% produce una reducción de $4 \%$ en el consumo per cápita. Por lo tanto, para obtener una reducción del $29 \%$ como la obtenida con la prohibición de fumar en el lugar de trabajo, sería necesario aumentar el precio de los cigarrillos en $73 \%$. Si se prohibiera fumar en todos los lugares de trabajo, se calcula que el consumo per cápita de la totalidad de la población disminuiría en $4,5 \%$ en los Estados Unidos y en 7,6\% en el Reino Unido, lo cual le supondría a la industria tabacalera pérdidas anuales de 1,7 mil millones de dólares estadounidenses y de 310 millones de libras esterlinas por disminución de las ventas. Esto explica la fuerte oposición de esta industria a las leyes que prohiben fumar en el lugar de trabajo.

En resumen, esta revisión sistemática revela que la prohibición de fumar en el lugar de trabajo no solo protege a los no fumadores de los peligros del consumo pasivo, sino que alienta a los fumadores a abandonar el consumo o, al menos, a reducirlo. El efecto combinado del abandono y la reducción del consumo reduce el consumo total de cigarrillos en $29 \%$, reducción que solo se podría obtener aumentando los impuestos sobre los cigarrillos en 73\%. (Fichtenberg CM, Glantz SA. Effect of smoke-free workplaces on smoking behaviour: systematic review. BMJ 2002;325:188-191).

\section{Método para comparar y combinar los estudios sobre los costos de las enfermedades}

Los estudios de costos de las enfermedades (ECE) calculan dos tipos de costos: directos, médi- cos y no médicos, asociados a la enfermedad, e indirectos, asociados a la pérdida de productividad debido a la morbilidad o a la mortalidad prematura. Al estudiar las repercusiones económicas de las enfermedades, a menudo se plantea el problema de cómo comparar o combinar las estimaciones de diferentes ECE. Este artículo describe un método para comparar y combinar los resultados de varios ECE e ilustra todos sus pasos con un ejemplo referido a las enfermedades cardiovasculares.

El método propuesto consta de los siete pasos que se detallan a continuación:

1) Identificación del diseño del estudio. El primer paso consiste en identificar el diseño de los ECE, puesto que no es aconsejable comparar o combinar estimaciones de costos obtenidas en ECE con diseños diferentes. Las principales características determinantes del diseño de un ECE son el método de estimación, los factores de ajuste, el período de tiempo estudiado y la perspectiva del estudio.

Los dos métodos de estimación más usados en los ECE son el del capital humano y el de la disposición a pagar. Los resultados obtenidos con uno y otro no son comparables y las estimaciones del primero suelen ser inferiores a las del segundo. El método del capital humano estima los costos indirectos asociados a la enfermedad y a la muerte prematura en términos de pérdida de productividad (ingresos perdidos), excluyendo así los costos derivados del dolor y el sufrimiento, el tiempo libre y el trabajo en régimen de voluntariado. El método de la disposición a pagar, que considera la cantidad que las personas estarían dispuestas a pagar para reducir su riesgo de lesiones, enfermedades o muerte, es subjetivo y puede ser de difícil uso en niños y ancianos, debido a la complejidad de las preguntas que hay que hacer.

Son varios los factores de ajuste usados en la estimación de costos, en primer lugar una tasa de descuento de los ingresos futuros, independiente de la inflación, para tener en cuenta que un ingreso futuro de determinada cantidad tendrá menos valor que un ingreso actual de la misma cantidad, por el simple hecho de que este puede ser invertido, aumentando su valor con el tiempo. Generalmente se usa una tasa de $5 \%$, aunque su valor puede oscilar entre el 2 y 10\%. El valor presente (PV) de un valor futuro (FV) al cabo de $t$ años, con una tasa anual de descuento $r$, se calcula con la fórmula: $\mathrm{PV}=\mathrm{FV}[1 /(1+\mathrm{r}) \mathrm{t}]$. Otro factor de ajuste son las tasas de inflación de los costos de años pasados. El valor del año base (BYV) de un valor de un año pasado (PYV) se calcula con los índices de precios al consumo del año base (BCPI) y del año pasado, con la fórmula: BYV = PYV [BCPI/PCPI]. La incapacidad también debe ser ponderada, dado que un día de incapacidad no significa necesariamente que ese 
día se haya tenido que renunciar a todas las actividades. Dicha ponderación puede ser, por ejemplo, la siguiente: imposibilidad de realizar una actividad fundamental, 0,5; restricción en la realización de una actividad fundamental, 0,4, y restricción en la realización de una actividad menor, 0,3.

Diferentes estudios pueden utilizar diferentes períodos de tiempo para estimar los costos. En este aspecto existen dos grandes modelos: uno basado en la prevalencia (generalmente anual) y el otro en la incidencia (toda la vida). Por su sencillez, se utiliza más el primero.

El enfoque desde diferentes perspectivas también puede proporcionar estimaciones diferentes. Aunque existen varias perspectivas posibles (de la sociedad, del gobierno, del proveedor de la atención sanitaria, del negocio, del individuo), se recomienda que todos los estudios de costo-efectividad, costobeneficio y costo-utilidad deberían ser realizados desde la perspectiva de la sociedad, que considera los costos para todos los sectores de la sociedad.

2) Estratificación en función de los componentes del costo. Una vez que se ha comprobado que las diferentes estimaciones de costos proceden de estudios con diseños idénticos o similares, se puede pasar a la fase siguiente, de estratificación según los componentes del costo. Aquí se trata de determinar cuales son los tipos de costos incluidos en los diferentes ECE, pues aunque los estudios tengan el mismo diseño, las comparaciones no serán válidas si los componentes incluidos no son los mismos. Básicamente existen tres clases de costos - directos, indirectos e intangibles - cada una de las cuales se puede subdividir en componentes. Los costos directos son los recursos gastados en actividades de prevención o de atención sanitaria: hospitales y otras instituciones asistenciales, médicos y otros profesionales sanitarios, medicamentos y dispositivos, investigación sanitaria, administración, etc. Los indirectos son los recursos que han sido sacrificados a consecuencia del trastorno de la salud y están relacionados con la pérdida de productividad debido a la incapacidad y a la mortalidad prematura. Por último, los intangibles son los costos del dolor, el sufrimiento, la ansiedad y la pérdida de tiempo de ocio, a los que se les asigna un valor monetario, generalmente mediante el método de la disposición a pagar.

3) Creación de componentes del costo concatenados. A continuación hay que crear una lista uniforme y mutuamente excluyente de componentes del costo concatenados de los diferentes ECE. Esta fase es fundamental para preparar los datos para el proceso de comparación, por lo que tiene que ser lo más objetiva que sea posible.

4) Ajuste según la inflación. Si los costos que hay que comparar no son del mismo año, para hacerlos comparables hay que considerar el efecto de la inflación o deflación, usando el índice de precios al consumo. Si los estudios a comparar se han efectuado en diferentes países con diferentes monedas, también hay que tener en cuenta las diferencias entre sus procesos inflacionarios y las fluctuaciones del cambio de divisas.

5) Ajuste según el crecimiento de la población. Además de la inflación, otro factor que puede influir sobre la validez de las comparaciones es el crecimiento de la población a lo largo del tiempo. El costo per cápita de cada componente del costo y el costo per cápita de todos los componentes del costo de los diferentes ECE se puede estimar aplicando las cifras de la población total en los años estudiados.

6) Comparación de las estimaciones del costo. Una vez que han sido ajustados en función de la inflación y del crecimiento de la población, los costos ya son directamente comparables y para ello se pueden usar métodos gráficos o de otros tipos, como la regresión, con el fin de determinar si las estimaciones de los diferentes estudios son homogéneas y constantes.

7) Combinación de las estimaciones del costo. Cuando se considere que las estimaciones del costo per cápita de determinados componentes del costo son razonablemente homogéneas y constantes a lo largo de los años, se pueden combinar las estimaciones del costo procedentes de diferentes ECE y tomar la media como la mejor estimación de los costos per cápita de dichos componentes del costo en un determinado año. Sin embargo, cuando otros componentes del costo muestren una tendencia temporal a lo largo de los años, se deben usar métodos de regresión para estimar los costos per cápita de esos otros componentes del costo en ese año. (Choi BCK, Pak AWP. A method for comparing and combining cost-of-illness studies: an example from cardiovascular disease. Chronic Dis Can 2002;23: 47-57) 